\section{SAT0564 SUBLINGUAL VACCINE: NEW CHALLENGE IN THE PREVENTION OF RECURRENT INFECTIONS IN AUTOIMMUNE DISEASES}

K. Llano Hernández ${ }^{1}$, S. Sanchez Ramón ${ }^{1}$, J. Ochoa Grullon ${ }^{1}$, P. Macarron ${ }^{2}$ C. Morado ${ }^{2}$, C. Martínez-Prada ${ }^{2}$, A. Rodríguez De La Peña ${ }^{1}$, E. Rodríguez Frías $^{1}$, M. Nuñez Beltran ${ }^{1}$, B. Fernández ${ }^{2}$, J.Á. Jover ${ }^{2}$, G. Candelas ${ }^{2}$. ${ }^{1}$ Inmunology; ${ }^{2}$ Rheumatology, Madrid Clinic Hospital, Madrid, Spain

Background: Disease-modifying antirheumatic drugs (DMARDs) and new biologicals have improved the prognosis of systemic autoimmune diseases (SAD), but reciprocally increase the risk of recurrent respiratory tract (RRTI) and urinary tract (RUTI) infections. Given the rising of antibiotic resistance, the search for new strategies for the prevention of recurrent infections is a priority.

Objectives: The purpose of this study was to evaluate the clinical benefit of the sublingual polybacterial vaccines on infections' rates in SAD patients.

Methods: A retrospective observational study on a cohort of SAD patients on active inmunosuppression with RRTI and RUTI was conducted. Patients were treated with multibacterial sublingual vaccine formulations either for RRTI (Staphylococcus spp., S. pneumoniae, K. pneumoniae, M. catarrhalis, $H$. influenzae) ${ }^{3}$ or/and RUTI (K. pneumoniae, E. coli, E. faecalis, P. vulgaris) ${ }^{4}\left(\right.$ Bactek/Uromune ${ }^{\circledR}$, Inmunotek SL Madrid, Spain) for 3-months period and clinical follow-up at 6- and 12-months. We monitored the frequency of infections, the intensity and severity of infections during follow-up. Immunological evaluation was performed, including: Serum immunoglobulin levels, IgG subclasses, specific antibodies' production: anti-pneumococcal, anti-Typhi polysaccharide and anti-tetanus toxoid antibodies, and $B$ and $T$ cell phenotype.

Results: A total of 50 patients were evaluated, and 34 were eligible at 12months. The mean age of the patients was $58 \pm 13$ years, 31 women $(91.17 \%)$ and 3 men $(8.82 \%), 44.11 \%(n=15)$ with rheumatoid arthritis (RA), $23.52 \%(n=8)$ with systemic lupus erythematosus (SLE), $8.82 \%(n=3)$ mixed connective tissue disease, 2.94\% $(n=1)$ ankylosing spondylitis, 2.94\% ( $n=1)$ sacroileitis, 2,94\% $(n=1)$ psoriatic arthritis, 2,94\% $(n=1)$ SLE/RA, 2.94\% $(n=1)$ discoid LE/Sjögren, 2.94\% ( $n=1)$ adult onset Still disease, 2,94\% $(n=1)$ sarcoidosis, $2.94 \%(n=1)$ SLElike. All patients showed a significant decrease in RRTI $(3.15 \pm 2.66$ vs $0.46 \pm 1.07$, $p<0.01)$ and RUTI $(1.85 \pm 2.49$ vs $0.35 \pm 1.06, p<0.01)$ frequency and use of antibiotics at 6-months of vaccine, except one with sarcoidosis. 23 of 34 patients $(67.64 \%)$ disclosed defects on specific antibody production to polysaccharide and protein immunization. Three patients with antibody production deficit and pneumonia required prophylactic intravenous Ig. No adverse effects or SAD relapses were noted during the 1 -year observational period.

Conclusions: Mucosal vaccination in immunosuppressed patients due to SAD with recurrent infections resulted in lower rates of RRTIs and RUTIs with subsequent improvement in their quality of life. Our preliminary results need to be validated in controlled trials.

References:

[1] G. Avila, A. Alonso, A. Pluma, C. Diaz, R. Juvernau, M. Lüpez Lasanta, S. Marsal. FRI0252 Risk Analysis for Recurrent Infectionsduring Biological Therapy in Chronic Inflammatory Arthritis. Ann Rheum Dis 2014;73:474-475 doi:10.1136/annrheumdis-2014-eular.5736.

[2] Sánchez-Ramón S, de Diego RP, Dieli-Crimi R, Subiza JL. Extending the clinical horizons of mucosal bacterial vaccines: current evidence and future prospects. Curr Drug Targets. 2014;15(12):1132-43.

Disclosure of Interest: None declared

DOI: 10.1136/annrheumdis-2017-eular.4802

\section{SAT0565 THE FREQUENCY OF SEPTIC ARTHRITIS AFTER ARTHROCENTESIS AND INTRA ARTICULAR GLUCOCORTICOID INJECTION IS LOW}

S.K. Petersen, R.A. Andreasen, I.M.J. Hansen. Rheumatology, Odense University Hospital, Svendborg Hospital, Svendborg, Denmark

Background: Intraarticular (IA) procedures have both diagnostic and therapeutic purposes in patients with arthritis. The therapeutic benefit of IA glucocorticoids (GC) injection in patients with rheumatologic diseases is well described. However, IA procedures are associated with increased risk of Septic arthritis (SA). Rapid diagnose and correct treatment is crucial to avoid joint damage, sepsis and potential fatal outcome. However, patients in risk of evolving SA secondarily to GC injection or arthrocentesis are not well defined.

Objectives: The aim of this study was to evaluate the risk of SA in patients who received an IA GC injection or an isolated joint puncture, and to describe possible characteristics for these patients.

Methods: All patients' undergoing IA procedures at the orthopaedic and rheumatological departments of Fuhnen from January 2006 to December 2013 were identified in the central database and included by register extraction. Patients who developed SA within 30 days after IA GC injection were registered as cases. SA was defined as clinically inflamed joint and positive synovial fluid culture. Retrospectively, data on age, gender, affected joint location, bacterial agent, pre-existing inflammatory disorder and death within 30 days were extracted from the patient files.

According to local recommendations a non-touch sterile technic where used for IA procedures. Patients were informed about the risk for SA and motivated to seek medical attention if suspicion of infection or lack of improvement.
Results: 22370 IA procedures were registered; 14118 IA GC injections and 8252 arthrocentesis. Eleven patients with SA were registered.

Eleven patients developed SA subsequently to IA GC injection $(0.08 \%$ of all GC injections). For patients' demography, joint distribution, bacterial agent and pre-excisting joint disease (Table 1). One patient died within 30 days after IA GC injection.

\begin{tabular}{lccccc}
\hline Sex & Age & Joint & Bacterial agent & Inflammatory Disease & Death, 30 days \\
\hline M & 83 & Shoulder & Grp. A streptococcus & No & + \\
M & 53 & Elbow & S. Aureus & No & - \\
M & 55 & Ancle & S. Aureus & Gout & - \\
M & 67 & Knee & Grp. A streptococcus & No & - \\
F & 83 & Knee & E. Faecalis & No & - \\
M & 58 & Knee & S. Aureus & No & - \\
M & 73 & Knee & S. Aureus & Gout & - \\
F & 50 & Shoulder & Grp. A streptococcus & RA & - \\
F & 66 & Knee & S. Aureus & No & - \\
F & 80 & Knee & S. Aureus & RA & - \\
M & 73 & Elbow & E. coli & RA & - \\
\hline
\end{tabular}

Conclusions: This study demonstrates that IA procedures can be performed with little risk of SA. The risk factors identified i.e. elderly patients with inflammatory joint diseases are consistent with those described in the literature [1].

We consider joint puncture technique and patient information for being essential when doing IA procedures. However, if SA occurs it is potentially fatal and therefore GC injection should be preserved for doctors with experience in joint diseases.

References:

[1] RA Andreasen et al. Prognostic factors associated with mortality in patients with septic arthritis: a descriptive study. Scand J Rheumatoly 2016, 1-6.

Disclosure of Interest: None declared

DOI: 10.1136/annrheumdis-2017-eular.1333

\section{SAT0566 ELECTRONEUROGRAPHIC FINDINGS IN PATIENTS WITH SUBACUTE/CHRONIC ARTICULAR SYMPTOMS OF CHIKUNGUNYA FEVER AND NEUROPATHIC COMPLAINTS - PRELIMINARY RESULTS}

L.F. da Rocha $\mathrm{Jr}^{1}{ }^{1,2}$, H.D. de Lima ${ }^{1}$, R.M. Correia ${ }^{1}$, M.R.D.A. Freitas ${ }^{1}$, P.R.S. de Melo ${ }^{1}$, A.G.L. de Mattos ${ }^{1}$, A.F.R. de Oliveira ${ }^{1}$, C.D.L. Marques ${ }^{2}$,

A.L.B.P. Duarte ${ }^{2}$, O. Lins ${ }^{1}$, A. Ranzolin ${ }^{1,2}{ }^{1}$ Instituto de Medicina Integral Professor Fernando Figueira; ${ }^{2}$ Hospital Das Clínicas of Pernambuco, Recife, Brazil

Background: The mechanisms of nonarticular pain associated with Chikungunya virus (CHIKV) infection are still poorly understood. Many patients that progress to Subacute/Chronic phases have neuropathic pain (NP) besides the articular symptoms. The NP is associated with a less favorable outcome, with greater impact on quality of life and lower efficacy of treatment. The NP can reflect a dysfunction of the nervous system, rather than a neurological lesion induced by CHIKV, but the pathophysiology of the neural disfunction is not completely understood. There are no studies evaluating the electroneurographic findings in patients with CHIKV infection and neuropathic symptoms.

Objectives: To evaluate the results of electroneurography (ENoG) of patients with Chikungunya Fever (CF) and neuropathic symptoms.

Methods: Patients with diagnosis of CF (clinical and epidemiological) and symptoms of paresthesias underwent ENMG of upper and lower limbs. The electrodiagnostic evaluation consisted of nerve conduction study of median, ulnar, tibial, fibular, sural and plantar nerves. Clinical and epidemiological data were also recorded.

Results: The sample was composed by 18 patients ( $82.3 \%$ females) with mean age of $56( \pm 9.9)$ years. The mean duration of symptoms of CF at the time of the ENoG was $23.8( \pm 10.8)$ weeks and the average of tender and swollen joints (including ankle and foot) was $29.6( \pm 21.5)$ and $9( \pm 9.9)$, respectively. The mean score of visual analogic scale (VAS) for pain was $4.4( \pm 2.4)$ and for fatigue was 5.9 $( \pm 2.9)$, considering values between 0 and 10 . No patient presented axial pain and the number of painful joints was higher in upper $(19.4 \pm 13.9)$ compared to lower

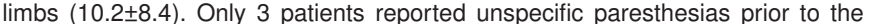
onset of arbovirosis and worsening after CF. However, these 3 patients had normal ENoG. Six patients had diabetes. Mononeuropathy was the most frequent result occurring in 12 subjects $(67 \%)$. Bilateral mononeuropathy of median nerve (at carpal tunnel) was found in 11 patients and one subject had median neuropathy just on the left hand. Other mononeuropathies were also present: bilateral tibial nerve in 4 , bilateral plantar nerve in 2 and bilateral fibular nerve in 1 patient. Distal axonal polyneuropathy was present in 8 cases ( 6 sensory and 2 sensorimotor); 5 of these were diabetic. The ENoG was normal in 4 cases. Ten patients were in use of prednisone (mean dose $11.4 \mathrm{mg} / \mathrm{d}$ ) and just 6 were using antineurophatic agents. Conclusions: Our preliminary results indicate that the ENoG is altered in most patients with chronic articular manifestations of CF and associated paresthesias. Mononeuropathy is the most commom finding, even in the chronic phase of the disease when the nonarticular edema is not common. Further clinical studies with a larger number of patients and follow-up tests will be needed to confirm our data. References:

[1] Andrade DC, Jean S, Clavelou P et al. Chronic pain associated with the 